\title{
Diagnostic Timelines and Self-reported Symptoms of Patients With Lung and Gastrointestinal Cancers Undergoing Radiation Therapy
}

Demetra Yannitsos ( $\nabla$ demetra.yannitsos@albertahealthservices.ca )

University of Calgary https://orcid.org/0000-0002-5515-8388

\section{Lisa Barbera}

University of Calgary

Abdullah Al-Rashdan

University of Calgary

\section{Petra Grendarova}

University of Calgary

\section{Research Article}

Keywords: patient-centered care, patient experience, symptom severity, oncology

Posted Date: April 16th, 2021

DOI: https://doi.org/10.21203/rs.3.rs-332873/v1

License: (c) (i) This work is licensed under a Creative Commons Attribution 4.0 International License. Read Full License 


\section{Abstract}

Background: Previous studies have found that patients with lung cancer report worse patient experience compared to other tumour groups. Reasons that may negatively impact patient experience include delays in diagnosis as well as inadequate symptom management. The purpose of this study was to compare the diagnostic timelines and symptom reports of patients with lung and gastrointestinal (GI) cancers.

Methods: This study included patients diagnosed with lung or GI cancers who attended a radiation oncology (RO) consultation and/or received radiation treatment between May and August 2019. Data collected included demographics, dates of diagnostic time points and self-reported symptom scores across 3 time points. A descriptive analysis was completed and the median number of days between time points were compared between tumour groups.

Results: Patients with lung cancer experienced a greater diagnostic delay compared to GI patients, specifically regarding the median number of days between the first investigative test and biopsy, with a difference of 21 days between tumour groups $(p<0.05)$. From RO consultation to the first treatment review appointment, $25 \%$ and $4 \%$ of lung and GI patients, respectively, reported worsening of symptoms. A greater proportion of lung patients reported worse symptoms scores during treatment compared to $\mathrm{GI}$ patients. This varied by specific symptom.

Conclusions: Delays in receiving a diagnosis and worse symptom experience during radiation treatment were demonstrated in this study and may indicate potential targets to improve patient experience.

\section{Introduction}

Understanding patient experience is necessary to provide high-quality, person-centered care and improve service delivery [1-3]. Patient experience is defined as "the sum of all interactions, shaped by an organization's culture that influences patient perceptions across the continuum of care" [4]. A seamless patient experience is integral to providing person-centered care, where the patient is an active partner in their cancer care $[5,6]$. Many programs aim to improve patient experience through quality improvement initiatives that identify gaps with validated measures and then apply strategies for bridging the gaps.

The Person-centered Radiation Oncology Service Enhancement (PROSE) is a programmatic multi-year radiation oncology department-wide initiative aiming to improve patient experience and the care patients receive at a comprehensive tertiary cancer centre. A baseline study completed by PROSE collected patient experience data within the radiation department [7]. Results of this study highlighted both system-level and tumour-group specific deficiencies that could be targeted for quality improvement (QI). One key finding was that patients with lung cancer consistently reported the least positive experience across their radiation therapy encounters compared to patients from other tumour groups, despite all oncology patients being exposed to the same clinical environment. The lung group reported the least positive experiences in all person-centered domains, including: access to care; information, communication \& education; patient preferences; physical comfort; emotional support; family \& friends; coordination of 
care; and, continuity \& transitions. Patients of certain tumour groups, including breast, prostate, and gastrointestinal $(\mathrm{Gl})$, reported the most favorable overall patient experiences across their radiation therapy encounters.

Various factors have been reported to influence the experience of patients with lung cancer. During time of diagnosis, factors associated with negative experiences include: an unexpected diagnosis (e.g. patients presenting only with a cough or are non-smokers) $[8,9]$, poor communication of the diagnosis from the oncologist, $[8,10]$ as well as the timeliness of diagnosis and receiving results [9]. Factors influencing patient experience during cancer therapies include feeling uninvolved in treatment decisions [10], lack of information regarding treatment options $[8,11,12]$ and inadequate management of side effects [8]. Patients also describe challenges in accessing supportive resources, including emotional support, indicating unmet supportive care needs for this population $[8,13]$.

Although certain factors of poor patient experience are identified in the literature, it is unclear why patients with lung cancer report less favorable patient experiences compared to other tumour groups within the same publicly funded system and department. The purpose of this study was to review and compare lung and GI patients' pretreatment diagnostic timelines and pre-and on-treatment symptom burden in order to understand if differences exist between tumour groups that might negatively affect experience. We hypothesized a greater diagnostic delay for lung patients compared to GI patients. We also anticipated a greater proportion of lung patients reporting higher symptom scores compared to $\mathrm{Gl}$ patients.

\section{Methods}

\section{Study population and design}

This was a retrospective study of consecutive lung and GI patients who had an initial radiation oncology consultation and/or at least one radiation treatment appointment at the Tom Baker Cancer Centre between May and August of 2019. This time frame was chosen to match the data collection time period of the PROSE baseline experience study, which identified poor lung cancer patient experience. The aim was to reflect the patient cohort of that study as closely as possible. Eligible patients include those with confirmation of lung or GI origin malignancy at any stage, and treatment intent. A total of 200 adult patients were included in this study, 100 lung and $100 \mathrm{GI}$ patients. GI was chosen as a comparison group to lung based on more positive experiences reported by GI patients in our previous patient experience study [7]. Patients diagnosed with GI cancers have similarities with lung cancer within our cancer care system, including baseline diagnostic testing requirements (need for endoscopic biopsies or advanced imaging techniques including PET Scan), prognosis, treatment techniques (e.g. use of concurrent chemoradiation in locally and regionally advanced disease), interdisciplinary approach, as well as occurrence in both sexes. Patients were excluded if they were scheduled but did not start their radiation course. This study was approved by institutional review board as part of PROSE program (protocol identifying number: HREBA.CC-18-0588). 


\section{Data collection}

Relevant variables and clinical time points were discussed and agreed upon by the study team, who consisted of oncology specialists and health services researchers. An abstraction form was created to collect relevant data.

Patient data were accessed from the electronic medical records (EMR). Variables included the patient's demographics (age and sex), tumor characteristics (stage, new primary vs recurrence), work-up characteristics (test types, date of first and last diagnostic test, referral and consultation appointment dates), and treatment characteristics (intent and radiotherapy dose/fractionation and delivery dates).

The dates of important time points included: date of biopsy, first diagnostic test, last staging investigation, referral sending and receiving dates by the cancer centre, first consultation and first radiation oncology consultation and the first and last radiation treatment. Treatment intent was coded according to the EMR data recorded by a treating radiation oncologist and verified by the study team radiation oncologist according to a specific treatment regimen.

Patients' self-reported symptoms from the revised Edmonton Symptom Assessment Scale (ESAS-r) [14] were retrieved. The ESAS-r is a patient-reported outcome measure (PROM) routinely collected in the radiation department at initial consultation, and at first and last treatment review appointments. The ESAS-r includes nine of the most common cancer symptoms, with 0 indicating patients do not experience the symptom to 10 indicating the symptom is at its worst $[15,16]$. We abstracted ESAS-r data that patients had completed at their consultation, first and last treatment reviews, as part of their routine care.

Data abstractors (DY) collected data from the EMR. A reviewer with clinical expertise in both tumor groups (PG) abstracted a sample of the data to ensure accuracy. Discrepancies in data were discussed until an agreement was reached.

\section{Analysis}

We completed a descriptive analysis and calculated the number of days between several time points within our data set, which were compared between lung and GI tumour groups. The time intervals analyzed included: first investigation to biopsy; biopsy to referral received; referral received to RO consultation; first investigation to RO consultation; and RO consultation to treatment start date. The median number of days between time points were calculated and compared using the Mann Whitney $U$ test. A regression model was also performed on the number of days between the first investigative test and RO consultation by tumour group and cancer phase. All statistical tests were performed with a significance level of $5 \%$.

For ESAS-r symptom data, an algorithm was used to calculate symptom complexity scores for each patient at each time point [17]. This approach to grouping patients by symptom severity has been used operationally in our organization to gauge symptom burden in patient populations and clinics. The algorithm provides a complexity score of high, medium or low based on ESAS-r responses. High scores 
are assigned if a patient reports a score of 10 on any symptom; scores either pain or 3-5 symptoms between 7 and 9; or reports 6 or more symptoms between 4 and 6 . A Medium symptom complexity score indicates a patient reported 1-2 symptoms with a score between 7 and 9 or reported 3-5 symptoms with a score between 4 and 6 . Patients that do not trigger a High or Medium score are assigned Low symptom complexity score. Changes in symptom complexity scores were compared at two different time frames: consultation to first treatment review, and first treatment review to last treatment review. A patient was assigned 'improvement' if their symptom score improved at the second time point (i.e. high to medium or low; medium to low). A patient was assigned 'worsening' if their symptom score became more severe (i.e. low to medium or high, medium to high). 'No change' was assigned to patients who had no change in complexity score between time points. Missing data, where the patient did not complete ESAS-r questions, was documented as not completed. Changes in symptom scores were only analyzed for patients that completed ESAS-r questions at both time points being compared.

The proportions of patients who reported medium-high individual symptom scores on each of the 9 ESAS-r symptoms were also calculated and compared across all appointments and for each tumour group. Scores $\geq 4$ were considered medium-high scores and included in the numerator [17].

\section{Results}

Within our study population, patients with lung cancer were older than patients with GI cancer. More patients with lung cancer were diagnosed with recurrence as opposed to a new diagnosis, with $17 \%$ more GI patients receiving a new diagnosis (Table 1). Most lung (43\%) and GI (35\%) patients were diagnosed with stage 4 cancer. GI patients with liver cancer were staged according to the Barcelona Clinic Liver Cancer staging system, however due to small numbers of diagnoses, we reported these as combined [18]. There was no difference in intent of treatment between the two groups, with over half of lung (51\%) and $\mathrm{GI}(54 \%)$ patients treated with curative intent. 


\section{Table I. Study Population Cancer Characteristics}

\begin{tabular}{llll|} 
Cancer Characteristics & $\begin{array}{l}\text { Lung } \\
(\mathbf{n = 1 0 0 )}\end{array}$ & $\begin{array}{l}\text { GI } \\
(\mathbf{n}=100)\end{array}$ & $\begin{array}{l}\text { P-value across tumour } \\
\text { group }\end{array}$ \\
\hline Age (years), mean (SD) & $\begin{array}{l}70.3 \\
(9.37)\end{array}$ & $\begin{array}{l}66.2 \\
(11.58)\end{array}$ & $0.006^{*}$ \\
\hline
\end{tabular}

Sex

\begin{tabular}{lccc} 
Male & 45 & 67 & $0.003^{*}$ \\
Female & 55 & 33 & \\
Phase of Cancer & & & $0.023^{*}$ \\
\cline { 1 - 3 } New diagnosis & 47 & 64 & \\
$\begin{array}{l}\text { Progression/new } \\
\text { metastases/recurrence }\end{array}$ & 53 & 36 &
\end{tabular}

\section{Cancer Stage}

\begin{tabular}{|c|c|c|c|}
\hline 1 & 12 & 4 & $<0.001$ \\
\hline 2 & 9 & 21 & \\
\hline 3 & 15 & 35 & \\
\hline 4 & 43 & 29 & \\
\hline Limited - Small cell lung & 6 & $\mathrm{n} / \mathrm{a}$ & \\
\hline Extensive - Small cell lung & 5 & $\mathrm{n} / \mathrm{a}$ & \\
\hline Liver & $\mathrm{n} / \mathrm{a}$ & 4 & \\
\hline Not stated & 10 & 7 & \\
\hline \multicolumn{4}{|l|}{ Treatment Intent } \\
\hline Curative & 51 & 54 & \multirow[t]{3}{*}{0.306} \\
\hline Palliative & 46 & 46 & \\
\hline Prophylactic & 3 & 0 & \\
\hline
\end{tabular}

*Statistically significant difference between lung and GI tumour groups

\section{Diagnostic time points}

Between the time of the first investigation and the biopsy, patients with $\mathrm{Gl}$ and lung cancer had a median of 0 days and 21 days, respectively $(p<0.001)$. The median number of days from biopsy to a referral sent to the cancer centre was double for GI (14 days) compared to lung (7 days) (p 0.002), with $18.3 \%$ more 
lung patients referred within 2 weeks compared to GI. The median number of days between the referral received by the cancer center and the RO consultation was approximately 2 weeks for both $\mathrm{GI}$ and lung groups. The time interval between the first investigation to the cancer centre referral showed a difference of 13 median days between tumour groups, significantly longer for lung patients ( $p$ 0.011) with approximately $25 \%$ of lung patients waiting $>56$ days between their first investigation and referral (Fig. 1).

Between the first investigation and the RO consultation, the median number of days was higher for patients with $\mathrm{Gl}$ cancer (40 days) compared to lung (33 days, $\mathrm{p}<0.001$ ). However, when the data was further divided by cancer phase for each tumour group (new diagnosis vs.

recurrence/progression/metastases), patients with an initial lung diagnosis encountered the longest period of time between first investigation and RO consultation, with a median of 66 days compared to 41 days for GI. Patients with recurrence/progression/metastases of their lung cancer encountered a median of 19 days compared to 36 days for $\mathrm{Gl}$. A linear regression model of the number of days between first investigation and RO consultation by tumour group and cancer phase was significant $(F=18.62, p<$ 0.001 , adjusted $R^{2} 0.2095$ ), with both the variables (tumour group and cancer phase) being statistically significant $(p<0.001)$.

Between the time from the initial RO consultation to treatment start, $56 \%$ of lung and $44 \%$ of GI patients started treatment within 2 weeks (Fig. 1). The median number of days were not statistically significant across tumour groups.

\section{Symptom Complexity Scores}

At initial RO consultations, symptom complexity scores were not statistically significant between tumour groups based on $95 \%$ confidence intervals (Table 2). However, a slightly higher proportion of patients with Gl cancer reported high symptom complexity scores (34.7\%) compared to lung (29.5\%) at RO consultations. There were $15.5 \%$ more lung patients reporting medium symptom scores compared to the GI group, and $10.3 \%$ more GI patients reporting low scores during consultations. At first treatment reviews, a higher proportion of patients with lung cancer reported high symptom complexity scores (41.5\%) compared to $\mathrm{GI}(24.1 \%)$, a difference of $17.4 \%$, and $21.5 \%$ more $\mathrm{GI}$ patients reported low scores compared to lung. For last treatment reviews, $15.1 \%$ and $19.7 \%$ more lung patients reported high and medium symptom complexity scores, respectively, compared to Gl, however, differences were not statistically significant. 
Table II. Proportion of symptom complexity scores at each time point

Symptom Complexity Scores (\%)

\begin{tabular}{|c|c|c|c|c|}
\hline Time Point & $\begin{array}{l}\text { Tumour } \\
\text { Group }\end{array}$ & Low [95\% Cl] & Medium [95\% Cl] & High [95\% Cl] \\
\hline \multirow[t]{2}{*}{ RO consultation } & Lung $(\mathrm{n}=61)$ & $\begin{array}{l}32.8[21.31 \\
46.00]\end{array}$ & $\begin{array}{l}37.7[25.61, \\
51.04]\end{array}$ & $\begin{array}{l}29.5[18.52 \\
42.57]\end{array}$ \\
\hline & $\mathrm{GI}(\mathrm{n}=72)$ & $\begin{array}{l}43.1[31.43, \\
55.27]\end{array}$ & $\begin{array}{l}22.2[13.27 \\
33.56]\end{array}$ & $\begin{array}{l}34.7[23.88 \\
46.86]\end{array}$ \\
\hline \multirow[t]{2}{*}{$\begin{array}{l}\text { First treatment } \\
\text { review }\end{array}$} & Lung $(n=41)$ & $\begin{array}{l}34.1[20.08 \\
50.59]\end{array}$ & $\begin{array}{l}24.4[12.36 \\
40.30]\end{array}$ & $\begin{array}{l}41.5[26.32, \\
57.89]\end{array}$ \\
\hline & $\mathrm{GI}(\mathrm{n}=54)$ & $\begin{array}{l}55.6[41.40, \\
69.08]\end{array}$ & $\begin{array}{l}20.4[10.63 \\
33.53]\end{array}$ & $\begin{array}{l}24.1[13.49, \\
37.64]\end{array}$ \\
\hline \multirow[t]{2}{*}{$\begin{array}{l}\text { Last treatment } \\
\text { review }\end{array}$} & Lung $(\mathrm{n}=22)$ & $\begin{array}{l}31.8[13.86, \\
54.87]\end{array}$ & $\begin{array}{l}36.4[17.20, \\
59.34]\end{array}$ & $\begin{array}{l}31.8[13.87, \\
54.87]\end{array}$ \\
\hline & $\mathrm{GI}(\mathrm{n}=24)$ & $\begin{array}{l}66.7 \text { [44.68, } \\
84.37]\end{array}$ & $16.7[4.74,37.38]$ & $16.7[4.74,37.38]$ \\
\hline
\end{tabular}

Changes in symptom complexity scores are documented in Table 3. From RO consultations to the first treatment reviews, $35.4 \%$ of patients with $\mathrm{Gl}$ cancer reported an improvement in symptoms compared to $19.4 \%$ of lung patients. The greatest difference between tumour groups was regarding worsening symptoms, as $25 \%$ of lung patients reported a worsening of symptoms compared to only $4.2 \%$ of $\mathrm{Gl}$, a difference of $20.8 \%$. No change in symptoms was reported for $55.5 \%$ of lung and $60.4 \%$ of GI patients. From the first to last treatment review appointment, a larger proportion of lung (68.4\%) and GI (63.6\%) patients reported no change in symptoms.

Table III. Changes in symptom complexity scores by time frames

\begin{tabular}{|c|c|c|c|c|}
\hline \multirow[t]{2}{*}{ Time Frames } & \multirow[t]{2}{*}{$\begin{array}{l}\text { Tumour } \\
\text { Group }\end{array}$} & $\begin{array}{l}\text { Improvement of } \\
\text { symptoms (\%) }\end{array}$ & \multirow[t]{2}{*}{$\begin{array}{l}\text { Worsening of } \\
\text { symptoms (\%) [95\% } \\
\text { Cl] }\end{array}$} & \multirow[t]{2}{*}{$\begin{array}{l}\text { No change of } \\
\text { symptoms (\%) [95\% } \\
\text { Cl] }\end{array}$} \\
\hline & & {$[95 \% \mathrm{Cl}]$} & & \\
\hline \multirow[t]{2}{*}{$\begin{array}{l}\text { RO consult to first } \\
\text { treatment review }\end{array}$} & $\begin{array}{l}\text { Lung } \\
(\mathrm{n}=36)\end{array}$ & $19.4[8.19,36.02]$ & $25.0[12.12,42.40]$ & $55.5[38.10,72.06]$ \\
\hline & $\begin{array}{l}\mathrm{GI}(\mathrm{n}= \\
48)\end{array}$ & $\begin{array}{l}35.4[22.16 \\
50.54]\end{array}$ & $4.2[0.51,14.25]$ & $60.4[45.27,74.23]$ \\
\hline \multirow[t]{2}{*}{$\begin{array}{l}\text { First to last } \\
\text { treatment review }\end{array}$} & $\begin{array}{l}\text { Lung } \\
(n=19)\end{array}$ & $15.8[3.38,39.58]$ & $15.8[3.38,39.58]$ & $68.4[43.45,87.42]$ \\
\hline & $\begin{array}{l}\mathrm{GI}(\mathrm{n}= \\
22)\end{array}$ & $18.2[5.19,40.28]$ & $18.2[5.19,40.28]$ & $63.6[40.66,82.80]$ \\
\hline
\end{tabular}


The proportion of patients who report medium-high scores for individual ESAS symptoms at each appointment are shown in Fig. 2. Relatively stable trends were seen in symptoms including drowsiness and depression, with consistently higher proportions of lung patients reporting medium-high depression scores compared to GI. Both patients with lung and GI cancers reported tiredness as the most common symptom experienced, and worsening overtime. For patients with GI cancers, worsening symptoms also included lack of appetite, nausea and shortness of breath (specifically at last review). Worsening of wellbeing was evident in patients with lung cancers across all appointments. In contrast, pain typically peaked at the first treatment review and improved by the last treatment for patients of both tumour groups, although higher proportions of GI patients reported medium-high scores at all times compared to lung. Anxiety trends improved overtime as well, however higher proportions of lung patients consistently reported moderate-high anxiety scores overtime.

\section{Discussion}

This study quantifies the time between important diagnostic time points for patients with lung and GI cancers treated with radiation therapy as well as compares symptom scores between the two tumour groups over the course of their radiation consultations and therapy encounters. We found that patients with lung cancer experienced longer wait times before having a biopsy and receiving a diagnosis compared to patients with GI cancer. This longer time frame is consistent with prior reports and may in part be due to many symptoms of lung cancer present similar to non-malignant conditions such as chest infection[8,9]. The differences can also be partly explained by the nature of investigative testing for GI patients, such as endoscopies, where the first investigative test and biopsy are completed at the same time point. Our results support our hypothesis that patients with lung cancer would experience a greater delay in their diagnosis compared to GI patients, specifically to receive a confirmed diagnosis.

The median number of days from the first investigation to the initial RO consult was shorter for patients with lung cancer compared to GI by one week. To better understand these differences, we divided both tumour groups into two sub- groups: those presenting with a new diagnosis and those with recurrent/metastatic/progression of cancer. The results revealed that newly diagnosed patients with lung cancer had the longest time frame between their first investigation and RO consultation compared to other sub-groups. There was a difference of 25 median days between newly diagnosed patients with lung and $\mathrm{Gl}$ cancers. This further supports the finding that individuals who are newly diagnosed with lung cancer may face a diagnostic delay in confirming their diagnosis.

Once patients with lung cancer were in the cancer system, the time interval between their referral and initial consultation with a radiation oncologist was generally the same as the GI group, two weeks on average. National groups are working to determine wait time benchmarks for medical services, including radiation therapy. The Wait Times Alliance of Canada currently recommends a (non-tumour specific) maximum of 10 working days between referral and appointment with the radiation oncologist [18]. It is also recommended 10 working days between radiation consultation and first radiation treatment sessions [19]. Our results indicate just over half of lung (51.6\%) and GI (53.3\%) patients meet the 
benchmark timeline between their referral and RO consultation. A higher proportion of patients with lung cancer (56\%) meet the recommended benchmark for time between RO consultation and radiation treatment start, compared to $\mathrm{Gl}(44 \%)$, and thus the differences in experiences between the two tumour groups are unlikely explained by this timeline.

Our results indicate a greater proportion of patients with lung cancer reported high symptom scores during treatment compared to GI. These results support the second part of our hypothesis that a higher proportion of patients with lung cancer would report more severe symptom scores. Approximately $17 \%$ and $15 \%$ more lung patients reported high symptom scores at their first and last treatment review appointments, respectively, compared to GI. Further, our results are novel as we observed three different time points to compare symptom scores. We found between the consultation and first treatment review, $25 \%$ of lung patients experienced worsening symptoms compared to only $4 \%$ of $\mathrm{Gl}$. In our cohort, patients with $\mathrm{Gl}$ cancer more commonly reported moderate-severe scores on symptoms including pain, nausea and lack of appetite. For these types of symptoms, many GI patients will receive referrals to dieticians and receive medications to assist in early symptom management at their consultation. Although we see some increases in moderate-high scoring of certain symptoms from consultations to first treatment review, patients with GI cancer reported trends in improved well-being between these time points as well.

Our results indicate a need for improved support to patients with lung cancer, which may help improve their radiation treatment experience. Our study found a higher proportion of patients with lung cancer reported moderate-high symptom scores for depression, anxiety and overall well-being at each time point, as well as shortness of breath (except for last treatment review). The proportion of patients with lung cancer reporting moderate-high well-being scores (moderate-high scores reflecting worse well-being) consistently increased over time. Anxiety, depression and overall wellbeing can be complex symptoms to intervene on, and guidelines exist for managing symptoms of anxiety and depression in cancer patients $[20,21]$. However, many patients are known to decline psychological or social support even when offered, adding another level of complexity to symptom management [22-24]. As well, preventing or managing dyspnea can be challenging, especially in older patients with additional comorbidities such as COPD or heart disease. In our study, patients with lung cancer were older in age compared to Gl. Although we did not have comorbidity index data of our study population, the literature supports that lung patients typically have multiple comorbidities with worse health status $[25,26]$. Although there may be challenges with symptom interventions, patients with lung cancer may benefit from increased support which may help improve their experience.

Few studies have compared the experience of patients with lung cancer and patient experience with other tumour sites. Ayanian et al. compared overall ratings of the quality of cancer care for patients with lung and colorectal cancer [26]. Excellent ratings were reported in $44 \%$ of lung and $53 \%$ of patients with colorectal cancer. Similar to our study population, stage IV cancer was more common in lung $(41 \%)$ than patients with colorectal cancer (19\%) [26]. Worse ratings for quality of care were reported for those with worse health status. Halpern et al. noted patients with lung cancer reported lower general health status 
and less favorable patient experience scores compared to patients with breast and colorectal cancer [25], although specific reasons for differences in experience were not investigated in either study.

This study has several limitations. This study only compared lung to one other tumour group. Including different or additional comparison groups may have highlighted other important factors. Additionally, because this was real-world data and not part of a clinical trial, we acknowledge the extent of missing data regarding symptom complexity scores, especially comparing treatment time points including last review. Non-response during this time may reflect sicker patients who are not captured and we may be underestimating the symptom burden towards the end of treatment. Another limitation is that this study was conducted retrospectively for the same time period as our previous patient experience study within the radiation department, which collected experience data from patients anonymously, and we do not have experience measures for these same patients. Therefore, we were unable to construct models using experience measures as outcomes, limiting our ability to draw a definite connection between these observations and our experience observations. Our conclusions are therefore inferential. Given these limitations, the data collected for our time points and basic demographic information was complete and accurate as dates do not change and are not open to interpretation. Further, the symptom data we collected was documented by patients in real-time at their appointment, therefore accurately reflecting their symptoms experienced. A strength of this study includes having these data, including dates and real-time collection, incorporated into one dataset.

\section{Conclusion}

Various factors influence how patients experience their cancer care. This study indicates that patients with lung cancer entering the radiation department experience greater diagnostic delays compared to patients with gastrointestinal malignancies. Further, a higher proportion of patients with lung cancer report more severe symptom scores throughout treatment as well as a worsening of symptoms from their consultation appointment to their first treatment review appointment. These results will be used to direct future research and quality improvement work in the radiation department to improve patient experience of individuals with lung cancer.

\section{Declarations}

Acknowledgments This study was supported by the Person-centered Radiation Oncology Service Enhancement (PROSE) program.

Funding: Person-centered Radiation Oncology Service Enhancement (PROSE) program.

Conflicts of interest: N/A

Availability of data: To provide anonymity to study participants, ethics approval does not permit disseminations of raw data 
Code Availability: Analysis was performed using Microsoft Excel and RStudio v1.0.153

Authors' contributions: All authors contributed equally to the conceptualization of the manuscript; DY drafted the manuscript, while LB, AR and PG provided clinical input and editorial oversight. All authors provided critical appraisal of the manuscript and approve its submission.

Ethics approval: The Institutional Research Information Services Solution (IRISS) Health Research Ethics Board through the University of Calgary provided ethics approval: HREBA.CC-18-0588

Consent to participate: N/A

Consent for publication: N/A

\section{References}

1. Canadian Institute for Health Information. Patient Experience. ClHI. Published 2020. Accessed October 28 (2020) https://www.cihi.ca/en/patient-experience\%0D

2. Wolf J, Niederhauser V, Marshburn D, Lavela SL (2014) Defining patient experience. Patient Exp J 1(1):7-19. doi:10.35680/2372-0247.1004

3. Lines LM, Lepore M, Wiener JM (2015) Patient-centered, person-centered, and person-directed care: They are not the same. Med Care 53(7):561-563. doi:10.1097/MLR.0000000000000387

4. The Beryl Institute. Defining Patient Experience. The Beryl Institute. Accessed October 28 (2020) https://www.theberylinstitute.org/page/definingpatientexp

5. Biddy R, Griffin C, Johnson N, Al E. Person-Centered Care Guideline.; 2015

6. Ekman I, Swedberg K, Taft C et al (2011) Person-centered care - Ready for prime time. Eur J Cardiovasc Nurs 10(4):248-251. doi:10.1016/j.ejcnurse.2011.06.008

7. Yannitsos D, Grendarova P, Al-Rashdan A et al. Patient Experience in Radiation Oncology.; 2019

8. Fitch MI (2019) The changing face of lung cancer: Survivor perspectives on patient engagement. Asia-Pacific J Oncol Nurs 6(1):17-23. doi:10.4103/apjon.apjon-43-18

9. Tsianakas V, Robert G, Maben J, Richardson A, Dale C, Wiseman T (2012) Implementing patientcentred cancer care: Using experience-based co-design to improve patient experience in breast and lung cancer services. Support Care Cancer 20(11):2639-2647. doi:10.1007/s00520-012-1470-3

10. Dy SM, Janssen EM, Ferris A, Bridges JF (2017) Live, Learn, Pass It on. J Patient Exp 4(4):162-168. doi:10.1177/2374373517714451

11. Bravi F, Di Ruscio E, Frassoldati A et al (2018) Patient and health care professional perspectives: A case study of the lung cancer integrated care pathway. Int J Integr Care 18(4):1-13. doi:10.5334/ijic.3972

12. Mokhles S, Nuyttens JJME, de Mol M et al (2018) Treatment selection of early stage non-small cell lung cancer: The role of the patient in clinical decision making. BMC Cancer 18(1):1-10. doi:10.1186/s12885-018-3986-5 
13. Sanders SL, Bantum EO, Owen JE, Thornton AA, Stanton AL (2010) Supportive care needs in patients with lung cancer. Psychooncology 19(5):480-489. doi:10.1002/pon.1577

14. Cuthbert CA, Watson L, Xu Y, Boyne DJ, Hemmelgarn BR, Cheung WY (2019) Patient-reported outcomes in Alberta: Rationale, scope, and design of a database initiative. Curr Oncol 26(4):e503e509. doi:10.3747/co.26.4919

15. Watanabe S, Nekolaichuk C, Beaumont C, Mawani A (2009) The Edmonton symptom assessment system-what do patients think? Support Care Cancer 17(6):675-683. doi:10.1007/s00520-008-05221

16. Watanabe SM, Nekolaichuk C, Beaumont C, Johnson L, Myers J, Strasser F (2011) A multicenter study comparing two numerical versions of the Edmonton symptom assessment system in palliative care patients. J Pain Symptom Manage 41(2):456-468. doi:10.1016/j.jpainsymman.2010.04.020

17. Selby D, Cascella A, Gardiner K et al (2010) A Single Set of Numerical Cutpoints to Define Moderate and Severe Symptoms for the Edmonton Symptom Assessment System. J Pain Symptom Manage 39(2):241-249. doi:10.1016/j.jpainsymman.2009.06.010

18. Bruix J, Llovet JM (2009) Major achievements in hepatocellular carcinoma. Lancet 373(9664):614616. doi.org/10.1016/S0140-6736(09)60381-0

19. Wait Time Alliance. Cancer Care: Wait time benchmarks for cancer care. Wait Time Alliance. Published 2014. Accessed October 30 (2020) https://www.waittimealliance.ca/benchmarks/cancercare/

20. Howell D, Keshavarz H, Esplen MJ, Hack T, Hamel M, Howes J, Jones J, Li M, Manii D, McLeod D, Mayer C, Sellick S, Riahizadeh S, Noroozi H, Ali, M on behalf of the Cancer Journey Advisory Group of the Canadian Partnership Against Cancer (July 2015) A Pan Canadian Practice Guideline: Screening, Assessment and Care of Psychosocial Distress, Depression, and Anxiety in Adults with Cancer. Canadian Partnership Against Cancer and the Canadian Association of Psychosocial Oncology, Toronto

21. Li M, Kennedy EB, Byrne N, Gérin-Lajoie C, Katz MR, Keshavarz H, Sellick S, Green E. Management of Depression in Patients With Cancer: A Clinical Practice Guideline. J Oncol Pract. 2016 Aug;12(8):747-56. doi: 10.1200/JOP.2016.011072

22. Clover KA, Mitchell AJ, Britton B, Carter G (2015) Why do oncology outpatients who report emotional distress decline help? Psychooncology 24(7):812-818. doi:10.1002/pon.3729

23. Carolan CM, Smith A, Davies GR, Forbat L (2018) Seeking, accepting and declining help for emotional distress in cancer: A systematic review and thematic synthesis of qualitative evidence. Eur J Cancer Care (Engl) 27(2):1-26. doi:10.1111/ecc.12720

24. Mosher C, Joseph W, Hanna N et al (2014) Barriers to Mental Health Service Use and Preference for Addressing Emotional Concerns among Lung Cancer Patients. Psychooncology 23(7):812-819. doi:10.1002/pon.3488.Barriers

25. Halpern MT, Urato MP, Lines LM, Cohen JB, Arora NK, Kent EE (2018) Healthcare experience among older cancer survivors: Analysis of the SEER-CAHPS dataset. J Geriatr Oncol 9(3):194-203. 
doi:10.1016/j.jgo.2017.11.005

26. Ayanian JZ, Zaslavsky AM, Arora NK et al (2010) Patients' experiences with care for lung cancer and colorectal cancer: Findings from the cancer care outcomes research and surveillance consortium. $J$ Clin Oncol 28(27):4154-4161. doi:10.1200/JC0.2009.27.3268

\section{Figures}

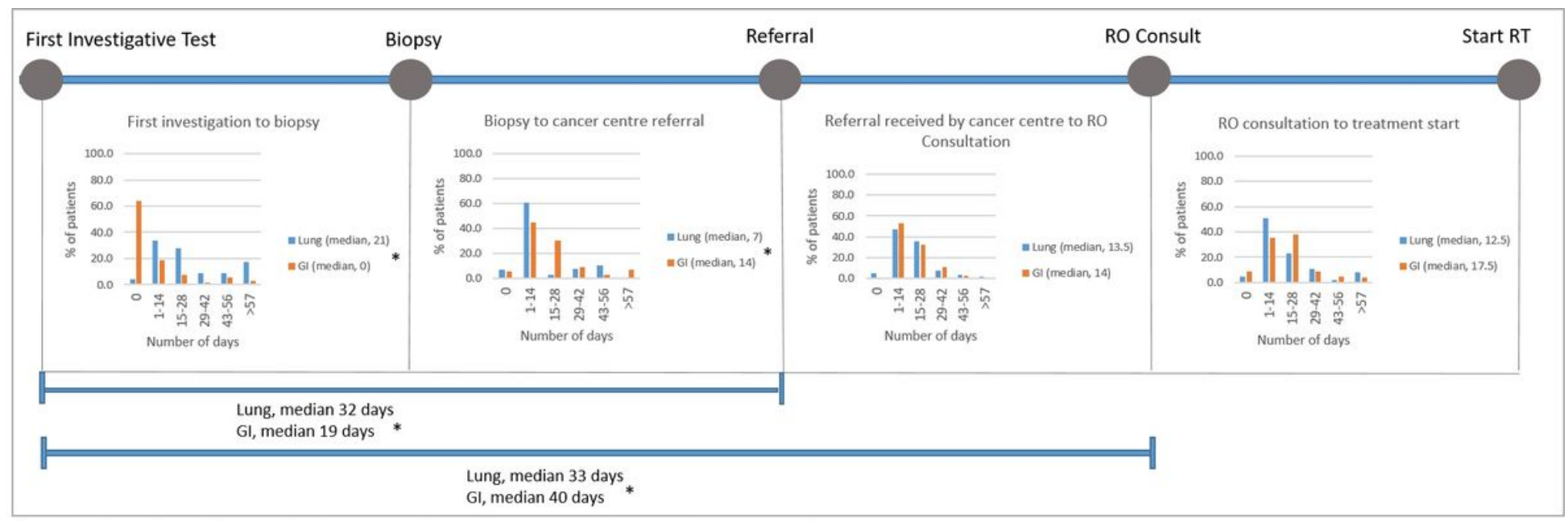

* Statistically significant difference of median number of days between lung and GI groups with P-value $<0.05$

\section{Figure 1}

Proportion of patients and the median number of days between time points. Proportions were calculated based on the number of patients in each tumour group and the number of days waited between each time point. The median number of days were statistically compared between tumour groups using the Mann Whitney U test. 


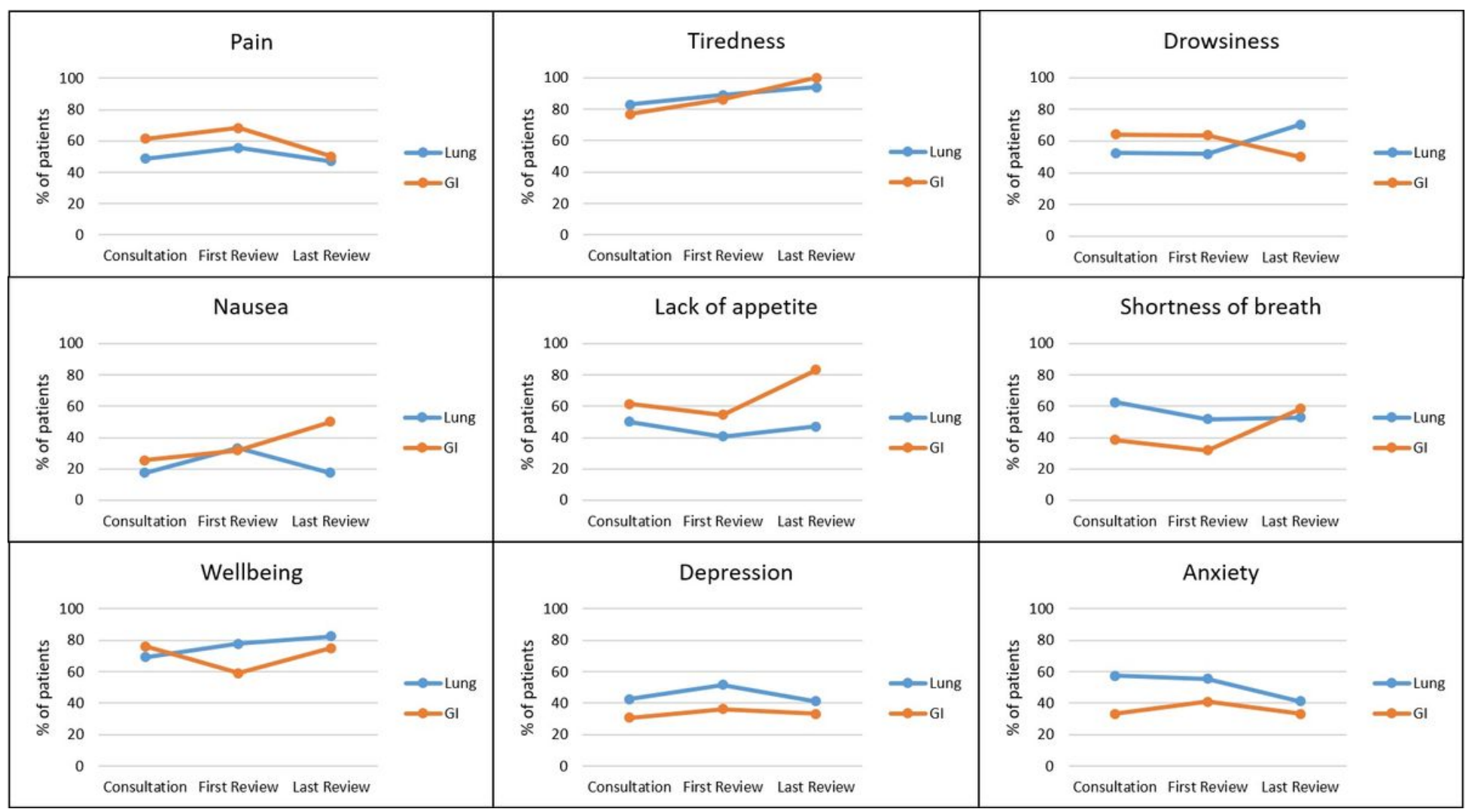

\section{Figure 2}

Proportion of patients reporting medium-high symptoms scores across appointments. Proportions were calculated for each time point (consult, first review and last review) and by tumour group (Lung and GI). Each symptom was scored on a scale from $0-10$. Scores $\geq 4$ are included in the numerator. Patients differ between time points. 